Serhii Hrybkov, Ph.D., Associate Professor, Department of Information Systems, National University of Food Technologies (Kyiv, Ukraine)

Olena Kharkianen, Ph.D., Associate Professor, Department of Information Systems, National University of Food Technologies (Kyiv, Ukraine)

Yuliya Gladka, Ph.D., Associate Professor, Department of Computer Mathematics and Information Security, Kyiv National Economic University named after Vadym Hetman (Kyiv, Ukraine)

\title{
FINDING THE BEST VERSIONS OF SCHEDULE FOR ORDER FULFILLMENT AT FOOD COMPANIES
}

\begin{abstract}
The article suggests a mathematical model of the multicriteria problem of formation of production tasks and operational-calendar plans at food companies, taking into account their specific features. The research was carried out, the bat algorithm and its modifications were adjusted and tested. The choice of the bat algorithm based on the Levy flight random search strategy is substantiated. This approach provides the formation of new and alternative plans for orders fulfillment at a food company, taking into account the proposed mathematical model.
\end{abstract}

Keywords: mathematical model, planning of the orders fulfillment, combined algorithms, the bat algorithm

Introduction. The food industry is one of the strategic ones in many countries. Food enterprises produce vital goods for the population. Fierce competition in domestic and foreign markets, the availability of cheaper imported products makes a food company apply modern methods of production management. Long-term planning does not allow responding quickly to the needs of consumers, which in turn leads to losses of market segments.

At the same time, the demand for finished products varies depending on the socio-economic circumstances in the country. Consumers are constantly demanding new range, quality, cost, and physical and organoleptic characteristics of products. Meeting that demand requires the food industry to constantly improve and expand the range of products, create alternative recipes that include new or improved components, as well as to refine their own technological processes. 
The vast majority of food companies produce a wide range of products, which leads to additional requirements and restrictions on production. A change in the production plan can lead to changes in the use of raw materials, the need for human resources, changes or readjustments of production equipment, changes in the schedules of shipment of finished products. Under these conditions, the management structure of the company must provide flexible and responsive production.

Today, the so-called method of goal decomposition is used for making operational and calendar plans and in solving production problems. According to it, local problems are solved without taking into account all the factors or using them as constraints. This approach is used in enterprise management information systems. Most of them are aimed at supporting the management of production, resources, automation of planning, accounting, control and analysis of business operations at the enterprise. And inter-department communication is provided through the use of corporate databases and data warehouses.

Existing information systems usually are cost-intensive and require some adaptation / integration into a specific production. The implementation of a new information system often requires reengineering of business management processes. At that, problems may be solved without taking into account all the influences. The process of integration and implementation of the information system requires additional costs, and its use depends on highly qualified managers of different levels in the field of IT technologies. Therefore, the use of such systems may not always ensure the accuracy and objectivity of decisions, especially in conditions of uncertainty and risk.

The importance of decision-making tasks and their complexity at different levels of management necessitates the creation of information technology for decision-making based on modified heuristic and evolutionary methods and algorithms, which can be used as part of the decision support system (DSS) of the food company.

\section{Literature review and problem statement}

[Hrybkov et al., 2020, p. 62-73] offered the information technology to solve the problem of planning the orders fulfillment at food companies, which is based on a combination of the ant colony, gray wolves and genetic algorithms. The use of the proposed technology is envisaged in solving complex problems and may require large resource inputs.

In [Hrybkov et al., 2018, p. 13-24], the authors proposed a mathematical model of planning the orders fulfillment and applied the ant colony method for its solution. The use of the proposed algorithms is impossible without the use of information systems, so in 
[Hrybkov et al., 2018, p. 13-24] the structure of web-oriented DSS in planning the orders fulfillment is proposed, as well as the technologies for its practical implementation are considered. But the disadvantage of the proposed approach is its focus on service enterprises, which disregards restrictions on the amount of raw materials and packaging materials needed for the manufacture of food products, the shelf life of finished products.

In [Kharkianen et al., 2018, 77-87], the authors proposed the information technology for adjusting the structure of the product range by means of data mining. The developed technology is designed to provide a food company with information support in making management decisions to find reserves to reduce the total cost of production and generate additional income. The proposed technology does not provide for the scheduling of orders, and is aimed only at developing proposals for adjusting the structure of the product range.

The work [Santosh, Vinod 2015, 116-123] is devoted to the use of genetic algorithms to solve problems of optimization of management and planning of industrial processes, but does not consider the features of individual industries.

In [Boyko, Hrybkov 2019, 116-123], the structure of a fuzzy situational network for a decision support system is proposed, the application of which will increase the efficiency of management of organizational and technical (technological) systems that operate at significant time intervals.

In [Sobchak, Lutai, Fedorenko 2019, 53-62], the information technology is proposed to solve the problem of decision-making support in the re-profiling of production at virtual instrument-making enterprises, according to the market demand. But the issue of decision-making to determine the feasibility of manufacturing a particular type of product and the maximum allowable production and distribution of production resources is not resolved.

The study [Yang-Kuei Lin 2018, 603-624] presents a mathematical model that takes into account only some efficiency criteria when planning operations on various technological machines. But the considered method of branches and borders has drawbacks in solving problems for equipment used in parallel.

The analysis of the above references allows us to assert the relevance of the two problems for food companies:

- formalization and mathematical modeling of scheduling orders, which allows the company to get the maximum profit;

- selection and substantiation of the choice of modified algorithms for solving the problem of forming optimal variants of the order fulfillment schedule. 


\section{Development of a mathematical model for scheduling the orders fulfillment}

For the mathematical model of scheduling orders, we introduce the following symbols: $t$ is the start time of the schedule, which is indicated by the date and time to the minute;

$\Delta t$ is time required to fulfill all orders in minutes;

$(t+\Delta t)$ is a planned period the production schedule is calculated for;

$i$ - a pending order, it regulates one type of a product that must be manufactured for the period $(t+\Delta t)$;

$j$ is the number of the stage from the set of stages $\left(j\right.$ is $\left.\omega_{i}\right)$ for the $i$-th order, where $\omega_{\mathrm{i}}$ is the number of required stages of manufacturing the $i$-th order;

$l$ is the equipment number from the set of equipment $\left(\mathrm{j}\right.$ is $\sigma_{\mathrm{i}}$ ) for the $i$-th order, where $\sigma_{\mathrm{i}}$ is the number of equipment involved to perform all stages in manufacturing the $i$-th order;

$t_{i}$ is time of beginning of manufacturing the $i$-th order;

$\Delta_{t i j l}$ is time of execution of the $j$-th stage on the $l$-th equipment for manufacturing the $i$ -th order;

$P t_{i j l}$ is time required for the preparation of the $l$-th equipment for execution of the $j$-th stage for manufacturing the $i$-th order, can take a zero value, if the preparation is not required;

$\eta t_{i j l}$ is time for cleaning the equipment after the $j$-th stage of manufacturing the $i$-th order on the $l$-th equipment;

$o_{i j l}$ is a parameter that takes the value $\{0,1\}\left(o_{i j} l=1\right.$, if the $j$-th stage can be performed on the $l$-th equipment for the manufacture of products on the $i$-th order; otherwise $o_{i j l}=0$ );

$n$ is the total number of orders to be executed for the period $(t+\Delta t)$;

$\theta_{i}$ is a parameter that takes the value $\{0,1\}\left(\theta_{i}=1\right.$, if the $i$-th order is executed for the period $(t+\Delta t)$; otherwise $\theta i=0)$;

$s d_{i}(t+\Delta t)$ is a unit cost for the $i$-th order for the period $(t+\Delta t)$;

$v c_{i}(t+\Delta t)$ is fixed costs for manufacturing a unit of the production for the $i$-th order for the period $(t+\Delta t)$;

$v z_{i}(t+\Delta t)$ is change in the cost of manufacturing a unit of production for the $i$-th order for the period $(t+\Delta t)$;

$o p_{i}(t+\Delta t)$ is the volume of finished products that must be produced for the $i$-th order in the period $(t+\Delta t)$;

$g_{i}$ is the amount of fine specified in a contract, which must be reimbursed to the customer if the $\mathrm{dt}_{\mathrm{i}}$ term of the order is delayed; 
$\Psi_{i}$ is a factor that takes the value $\{0,1\}, \Psi_{i}=1$ if $\left(t_{i}+F_{2 i}(t+\Delta t)\right)<d t_{i}$, otherwise $\Psi_{i}=$ 0 , and determines the need to reimburse the customer if the $\mathrm{dt}_{\mathrm{i}}$ term is delayed;

$r_{i}$ is the list of resources required for the manufacture of products on the $i$-th order;

$\omega_{i}$ is the number of technological stages for execution of the $i$-th order;

$h k_{i}$ is a factor that takes into account the necessity to pay a fine for each day of delay;

$\sigma_{i}$ is the number of equipment used to perform all stages in manufacturing the $i$-th order;

$t o_{i j-1}$ is the time of transition / waiting between execution of the $j$-th stage to the $(j-1)$ stage;

$c_{i j l}$ is the cost for one hour in execution of the $j$-th stage on the $l$-th equipment for manufacturing products on the $i$-th order;

$c n_{i j l}$ is the cost of fulfillment or utilization of a unit of the received substandard production at execution of the $i$-th order on the $l$-th technological equipment;

$v n k_{i j l}$ is the total number of substandard products received for each unit of production during execution of the $j$-th stage of the $i$-th order on the $l$-th technological equipment;

$d t_{i}$ is time for which products under the $i$-th order should be manufactured;

$k$ is the type of raw material required to execute the $i$-th order, and its total amount required for execution of the $i$-th order $r_{i}$;

$r k_{i k}$ is the amount of raw material required for manufacturing a unit of production on the $i$-th order;

$r t_{i k}$ is time of receipt of the $k$-th component for execution of the $i$-th order;

$v s r_{i k}$ is the cost of storing the $k$-th component for execution of the $i$-th order.

We define the following partial criteria of efficiency of the optional plan for the food company:

1) The maximum profit obtained by fulfilling all orders for the planning period $(t+\Delta t)(1)$.

$$
F_{l}(t+\Delta t)=\sum_{i=1}^{n}\left(\theta_{i} *\left(s d_{i}(t+\Delta t)-\left(v c_{i}(t+\Delta t)+v z_{i}(t+\Delta t)\right)\right)\right) * o p_{i}(t+\Delta t) \rightarrow \max
$$

2) Minimization of production time of each $i$-th order for the period $(t+\Delta t)(2)$, as well as restrictions (3) and (4), which regulate the production time of each product for the $i$-th order and restrictions on the term of completion of manufacturing the production must not exceed a given period. 


$$
\begin{aligned}
& F_{2}(t+\Delta t)=\max _{i}\left(F_{2 i}(t+\Delta t)\right)=\max _{i}\left(\sum_{j=1}^{\omega_{i}} \sum_{l=1}^{\sigma_{i}}\left(o_{i j l} *\left(p t_{i j l}+t_{i j l}+\eta t_{i j l}+t o_{i j-1}\right)\right)\right) \rightarrow \min \\
& t \leq t_{i}+F_{2 i}(t+\Delta t) \leq t+\Delta t \\
& t \leq t_{i}-F_{2 i}(t+\Delta t) \leq d t_{i}
\end{aligned}
$$

3) Minimization of total fines for delayed execution of the order for the period $(t+\Delta t)(5)$

$$
F_{3}(t+\Delta t)=\sum_{i=1}^{n}\left(g_{i} * \psi_{i} * h k_{i}\right) \rightarrow \min
$$

4) Minimization of total costs during the passage of the entire technological process through the relevant technological areas when fulfilling orders for a given period $(t+\Delta t)(6)$.

$$
F_{4}(t+\Delta t)=\sum_{i=1}^{n}\left(\sum_{j=1}^{\omega_{i}} \sum_{l=1}^{\sigma_{i}}\left(\left(o_{i j l} *\left(p t_{i j l}+t_{i j l}+\eta t_{i j l}\right)\right) * c_{i j l}\right)\right) \rightarrow \text { min }
$$

5) Minimization of costs for fulfillment and utilization of the received substandard production at execution of all orders (7).

$$
F_{5}(t+\Delta t)=\sum_{i=1}^{n} \sum_{j=1}^{\omega_{i}} \sum_{l=1}^{\sigma_{i}}\left(o_{i j l} * c n_{i j l} * v n k_{i j l} * o p(t+\Delta t)\right) \rightarrow \min
$$

6) Minimization of costs for storage of finished products before the specified date of shipment (8).

$$
F_{6}(t+\Delta t)=\sum_{i=1}^{n}\left(v z_{i} * o p(t+\Delta t) * \max \left(0, d t_{i}-\left(t_{i}+F_{2 i}(t+\Delta t)\right)\right) \rightarrow \min \right.
$$

7) Minimization of costs for storage of raw materials necessary for the manufacture of products (9).

$$
F_{7}(t+\Delta t)=\sum_{i=1}^{n} \sum_{k=1}^{r_{i}}\left(v s r_{i k} * r k_{i k} * o p(t+\Delta t) * \max \left(0, t_{i}-r t_{i k}\right) \rightarrow \min \right.
$$

The overall evaluation function will be represented by an additive convolution of all criteria (10).

$$
F_{0}^{\prime}=\lambda_{1} F_{1}-\sum_{\gamma=3}^{7} \lambda_{\gamma} F_{\gamma}-\lambda_{8} F_{8} \rightarrow \max
$$

where $\lambda$ is the weight factor of the criterion $\lambda_{\gamma} \epsilon[0,1]$. 
Depending on the social and economic situation, as well as the experience of a manager who is responsible for making an operational and calendar schedule for execution of orders, the problem can be solved in different ways, namely:

- in the assessment of effectiveness of the operational and calendar schedule for execution of orders, all criteria are accepted, and they are ranked;

- the problem is simplified to selection of certain partial criteria.

The mathematical model is a combinatorial multi-criteria NP-complete problem. It includes a number of restrictions: the total volume of production should not be greater than the capacity of the enterprise for a given period; it is not possible to violate requirements and terms of storage of raw materials; it is not possible to violate requirements and terms of storage of finished products; for a certain period of time it is allowed to use each technological equipment for the manufacture of only one type of a product or a semi-finished product.

Based on review of the references we may conclude on expediency of use of the bat method and its modifications along with other metaheuristic population algorithms.

\section{The bat algorithm and its modifications}

The peculiarities of the bats behavior during hunting include the ability to actively gather information and make decisions very quickly. For orientation in space, bats use echolocation, they emit ultrasound, which reaches the victim and is reflected back. Most bat species have advanced echolocation tools that they use to detect a target and obstacles, as well as to be able to settle on the surface in the dark. Due to echolocation, the bat determines location of the victim and attacks it. The bat makes decisions about its actions in space very quickly based on information obtained from the external environment. One of the main advantages of the bat algorithm is the speed of execution and potentially more power than in the particle swarm algorithm. The algorithm may seem more complex than the most other swarm intelligence algorithms, however, it can be applied quite effectively to optimization problems and ensures the search for optimal results, spending less time [Suganthi, 2017].

The bat algorithm adheres to the following rules:

- all bats use echolocation to analyze the distance, as well as to distinguish between food and natural obstacles;

- bats move randomly at speed $v_{i}$ to a position $x_{i}$ with fixed frequency $f_{\min }$, variable wavelength $\lambda$ and volume $A_{0}$ to find the target; 
- bats can automatically adjust the wavelength (or frequency, since frequency = $1 /$ wavelength), pulse emission and pulse velocity $r_{i}[0,1]$, depending on the distance to a particular object or target;

- the volume rate varies from a more positive $A_{0}$ to a les constant value $A_{\min }$.

Given that the speed of sound in the air is approximately $300 \mathrm{~m} / \mathrm{s}$, the wavelength for sound with a constant frequency is determined by the formula (11).

$$
\lambda=\frac{v}{f}
$$

Bats use the delay from signal radiation to echo detection, the time difference in echo detection on two ears in order to build a three-dimensional model of the surrounding space. Using the constructed model, they detect the distance to the surrounding objects, the distance to the target, the type of the target, the speed of its movement. When doing so, they use the Doppler effect, which consists in changing the frequencies and wavelengths of radiation through the movement of a radiation source of the wave. If the wave source moves in the medium and emits waves, the distance between the waves depends on the speed and direction of movement of the source and receiver. If the source moves towards the receiver, i.e. catches up with the wave, the wavelength decreases and vice versa - if it moves in the direction opposite to the source, the length increases by the formula (12).

$$
\lambda=\frac{2 \pi(c-v)}{w_{0}}
$$

where $w_{0}$ is angular wave frequency;

$c$ is the speed of wave propagation in the medium;

$v$ is the speed of the sound source relative to the medium (with a sign " + " if the source approaches the receiver and with a sign "-" if it moves away).

The following values are used for the algorithm operation:

- wave frequencies are in the range $\left[f_{\min } ; f_{\max }\right]$;

- they correspond to the range of wavelengths $\left[\lambda_{\min } ; \lambda_{\max }\right]$.

Let us describe the motion of bats by formulas (13-15), which are necessary for the algorithm.

$$
\begin{aligned}
& f_{i}=f_{\text {min }}+\left(f_{\text {max }}-f_{\text {min }}\right) \beta \\
& v_{i}^{t}=v_{i}^{t-1}+\left(x_{i}^{t}-x_{*}\right) f_{i} \\
& x_{i}^{t}=x_{i}^{t-1}+v_{i}^{t}
\end{aligned}
$$

where $\beta \in[0,1]$ is an arbitrary value;

$x_{*}$ is the best current solution. 
The pulse amplitude is updated and emitted at each iteration of the algorithm. As the bat approaches the target, the volume of its pulses decreases (16), and the pulse frequency increases. (17).

$$
\begin{aligned}
& A_{i}^{t+1}=\alpha A_{i}^{t} \\
& r_{i}^{t+1}=r_{i}^{0}\left[1-\frac{1}{e^{\gamma t}}\right]
\end{aligned}
$$

where $\alpha$ and $\gamma$ are constant values.

The complexity of such an algorithm directly depends on the number of spices in the flock used in the algorithm, the number of extremes and the dimension of the problem.

The chaotic bat algorithm (CBA) is based on chaos theory using piecewise chaotic maps. Chaotic systems are very common in natural and social systems that have complex, random and accurate characteristics [Sha-ShaGuo, Jie-ShengWang, Xiao-XuMa 2019, Suganthi Jeyasingh 2017]. Chaotic motion is a very unstable motion in a deterministic system, limited by the space of finite phases. Chaos is a form of aperiodic motion that is unique and common in nonlinear systems. The given logistic equation is known as the most typical for chaotic systems (18).

$$
S_{k+1}=\mu S_{k}\left(1-S_{k}\right)
$$

where $\mu$ is the transition factor, which is a constant one,

$S_{k}$ is the factor value formed by the rule of a chaotic map, $\mathrm{S} \in(0,1)$. The sequence $\mathrm{S}_{0}$, $\mathrm{S}_{1}, \ldots$ is deterministic, which is formed according to a certain rule of a chaotic map. There are 10 typical patterns of chaotic mapping, but the optimal type of a chaotic map is selected by simulation experiments on test functions. Based on a number of scientific papers, the application of various chaotic maps was analyzed and the choice of the best piecewise chaotic map was proposed. The initial value of 0.7 is proposed, which provides the speed of finding the optimal solution.

The bat algorithm of the Levy flight search strategy (LBA) relies on the Levy flight method, which explores the flight trajectory of living beings in search of food in an uncertain and unpredictable environment. Using the Levy flight method is the best strategy for finding an object that has a random location in space, as well as when it is impossible to model the space mathematically.

The bat movement in this algorithm corresponds to the non-Gaussian stochastic process, performs a large number of jumps in space and repeatedly changes direction, which allows expanding the search space. 
Combined with the bat echolocation function, this significantly improves the efficiency of the bat algorithm. Therefore, the improved algorithm replaces equation (15) with equation (19).

$$
x_{i}^{t}=x_{i}^{t-1}+\operatorname{levy} x\left(x_{i}^{t-1}-x^{*}\right)+V_{i}^{t}
$$

The Levy flight is used by a single bat to find the optimal location, which provides increased iterations of checks and optimization in the global search process. It helps to avoid hitting the local optimum and speeds up finding the optimal solution.

The shrink factor bat algorithm (SBA) is used to avoid hitting the local optimum, which can impede finding the global optimum. To avoid this problem, it is advisable to use a reduction factor that provides population diversity and increases the efficiency of finding the optimum. To apply this approach, formula (19) is converted into a formula (25).

$$
\begin{aligned}
V_{i}^{t} & =V_{i}^{t-1}+\left(x_{i}^{t-1}-x_{*}\right) f_{i} * k \\
k & =\frac{2}{\mid 2-d-\sqrt{d-4 d \mid}}
\end{aligned}
$$

where $d$ is a constant that is selected depending on the problem.

The following algorithms are compared: the classical bat algorithm (BA), the bat algorithm of the Levy flight search strategy (LBA), and the shrink factor bat algorithm (SBA).

The efficiency of algorithms is evaluated on the basis of the following indicators: time to find the optimal schedule; the effectiveness of the found plan (defined as the deviation from the assessment of the actual plan by the objective function); reduction of the time of order execution (calculated as the difference between the actual and the proposed plan).

It should be noted that the time to find the optimal plan depends on the computer technology performance. The approbation was carried out on the basis of statistical data on the execution of orders for previous periods, so the actual plan was taken as one that was executed. 25, 50, 75 and 100 orders were randomly selected for different time periods. The studies were performed with the same parameters for each algorithm. Table 1 compares the application of algorithms for 50 orders. 
Table 1. Comparison of algorithms for 50 orders.

\begin{tabular}{|c|c|c|c|c|}
\hline № & $\begin{array}{c}\text { Method } \\
\text { name }\end{array}$ & $\begin{array}{c}\text { Time to find an } \\
\text { optimal plan, min }\end{array}$ & $\begin{array}{c}\text { Efficiency of the fined } \\
\text { optimal plan, cond. units }\end{array}$ & $\begin{array}{c}\text { Reduction of the time } \\
\text { of order execution, } \\
\text { hours }\end{array}$ \\
\hline 1 & BA & 16 & 1234 & 8 \\
\hline 2 & CBA & 18 & 1234 & 7 \\
\hline 3 & LBA & 8 & 1234 & 5 \\
\hline
\end{tabular}

According to the results of comparing the considered algorithms, the use of LBA is proposed. The disadvantages of this method include a large number of free parameters, the value of which often defines the result; on the other hand, there is no reason to choose these values. LBA is to use the Levy method for the optimum, which provides a quick search for large-scale problems. When the number of orders is less than 25 , all algorithms work almost the same, and when it is increased, there is an immediate need to use modified algorithms. It is the considered algorithms that make it possible to avoid local optimums.

Conclusions. According to the results of research:

- the mathematical model of scheduling the execution of orders is proposed taking into account the main features of activity of the food companies that allows them to estimate formation of optimum options of the order fulfillment schedule;

- a modified LBA algorithm is proposed, which provides the formation of new and alternative plans for order fulfillment, taking into account the developed mathematical model.

Application of these approaches as part of the information DSS would have a positive effect on the economic performance of the food business.

\section{References}

1. Boyko R. O., Hrybkov S. V. (2019) Network Structures in Management of the Complex Organizational and Technical (Technological) Systems. The Food Industry, 25. https://doi.org/10.24263/2225-2916-2019-25-17

2. Georgiadis G. P., Elekidis, A. P., Georgiadis, M. C. (2019) Optimization-Based Scheduling for the Process Industries: From Theory to Real-Life Industrial Applications. Processes. V. 7. https://doi.org/10.3390/pr7070438 
3. Hrybkov S., Kharkianen O., Lytvynov, Ovcharuk V., Ovcharuk I. (2020). Development of Information Technology for Planning Order Fulfillment at a Food Enterprise, 1/3. (103). https://doi.org/10.15587/1729-4061.2020.195455

4. Hrybkov S., Lytvynov V., Oliinyk H. (2018). Web-oriented decision support system for planning agreements execution. Eastern-European Journal of Enterprise Technologies, 3/2. (99). https://doi.org/10.15587/1729-4061.2018.132604

5. Kharkianen O., Myakshylo O., Hrybkov S., Kostikov M. (2018) Development Of Information Technology For Supporting The Process Of Adjustment Of The Food Enterprise Assortment. Eastern-European Journal of Enterprise Technologies, 1/3. (91). https://doi.org/10.15587/1729-4061.2018.123383

6. Santosh K. S., Vinod, K. G. (2015) Genetic Algorithms: Basic Concepts and Real World Applications. International Journal of Electrical, Electronics and Computer Systems (IJEECS), 3 (12).

7. Sha-Sha Guo, Jie-Sheng Wang, Xiao-Xu Ma (2019) Improved Bat Algorithm Based on Multipopulation Strategy of Island Model for Solving the Global Function Optimization Problem / Mode of access: https://www.hindawi.com/journals/cin/2019/ 6068743/. https://doi.org/10.1155/2019/6068743

8. Sobchak A., Lutai L., Fedorenko M. (2019) Development of Information Technology Elements for Decision-Making Support Aimed at Re-Structuring Production at Virtual Instrument-Making Enterprises. Eastern-European Journal of Enterprise Technologies, 5/4 (101). https://doi.org/10.15587/1729-4061.2019.182039

9. Suganthi Jeyasingh (2017) Modified Bat Algorithm for Feature Selection with the Wisconsin Diagnosis Breast Cancer (WDBC) Dataset / Mode of access: https://www.ncbi.nlm.nih.gov/pmc/articles/PMC5555532/.

10. Yang-Kuei Lin (2018) Scheduling Efficiency on Correlated Parallel Machine Scheduling Problems // Operational Research. Vol. 18, Issue 3. https://doi.org/10.1007/s12351-017-0355-0 